\title{
Correlated electron metal properties of the honeycomb ruthenate $\mathrm{Na}_{2} \mathrm{RuO}_{3}$
}

\author{
L. S. I. Veiga $\odot,{ }^{1,2}$ M. Etter, ${ }^{3}$ E. Cappelli, ${ }^{4}$ H. Jacobsen $\odot,{ }^{5,6}$ J. G. Vale,,${ }^{1}$ C. D. Dashwood, ${ }^{1}$ D. Le $\odot,{ }^{7}$ F. Baumberger, ${ }^{4,8}$ \\ D. F. McMorrow, ${ }^{1}$ and R. S. Perry ${ }^{9}$ \\ ${ }^{1}$ London Centre for Nanotechnology and Department of Physics and Astronomy, University College London, \\ Gower Street, London WC1E 6BT, United Kingdom \\ ${ }^{2}$ Diamond Light Source Ltd., Harwell Science \& Innovation Campus, Didcot, Oxfordshire OX11 ODE, United Kingdom \\ ${ }^{3}$ Deutsches Elektronen-Synchrotron (DESY), Hamburg 22607, Germany \\ ${ }^{4}$ Department of Quantum Matter Physics, University of Geneva, 24 Quai Ernest-Ansermet, 1211 Geneva 4, Switzerland \\ ${ }^{5}$ Clarendon Laboratory, Department of Physics, University of Oxford, Oxford OX1 3PU, United Kingdom \\ ${ }^{6}$ Paul Scherrer Institute, Laboratory for Neutron Scattering and Imaging, 5232 Villigen, Switzerland \\ ${ }^{7}$ ISIS Facility, Rutherford Appleton Laboratory, STFC, Chilton, Didcot OX11 0QX, United Kingdom \\ ${ }^{8}$ Swiss Light Source, Paul Scherrer Institute, CH-5232 Villigen, Switzerland \\ ${ }^{9}$ London Centre for Nanotechnology and Institute for Materials Discovery, University College London, \\ Gower Street, London WC1E 6BT, United Kingdom
}

(Received 23 April 2020; revised 16 July 2020; accepted 20 August 2020; published 14 September 2020)

\begin{abstract}
We report the synthesis and characterization of polycrystalline $\mathrm{Na}_{2} \mathrm{RuO}_{3}$, a layered material in which the $\mathrm{Ru}^{4+}\left(4 d^{4}\right.$ configuration) form a honeycomb lattice. The optimal synthesis condition was found to produce a nearly ordered $\mathrm{Na}_{2} \mathrm{RuO}_{3}(C 2 / c$ phase), as assessed from the refinement of the time-of-flight neutron powder diffraction. Magnetic susceptibility measurements reveal a large temperature-independent Pauli paramagnetism $\left[\chi_{0} \sim 1.42(2) \times 10^{-3} \mathrm{emu} / \mathrm{mol} \mathrm{Oe}\right]$ with no evidence of magnetic ordering down to $1.5 \mathrm{~K}$, and with an absence of dynamic magnetic correlations, as evidenced by neutron scattering spectroscopy. The intrinsic susceptibility $\left(\chi_{0}\right)$ together with the Sommerfeld coefficient of $\gamma=11.7(2) \mathrm{mJ} / \mathrm{Ru} \mathrm{mol} \mathrm{K}{ }^{2}$ estimated from heat capacity measurements gives an enhanced Wilson ratio of $R_{W} \approx 8.9(1)$, suggesting that magnetic correlations may be present in this material. While transport measurements on pressed pellets show nonmetallic behavior, photoemission spectroscopy indicates a small but finite density of states at the Fermi energy, suggesting that the bulk material is metallic. Except for resistivity measurements, which may have been compromised by near-surface and interface effects, all other probes indicate that $\mathrm{Na}_{2} \mathrm{RuO}_{3}$ is a moderately correlated electron metal. Our results thus stand in contrast to earlier reports that $\mathrm{Na}_{2} \mathrm{RuO}_{3}$ is an antiferromagnetic insulator at low temperatures.
\end{abstract}

DOI: 10.1103/PhysRevMaterials.4.094202

\section{INTRODUCTION}

The identification and characterization of new quantum materials remains one of the principal drivers of research into complex systems. Quantum materials exhibit a plethora of functional properties, from superconductivity to topological states, driven by the subtle interplay between competing energy scales $[1,2]$. The inherent instability of perturbation in these systems is both a blessing and curse; exotic ground states can be stabilized out of the competition, but these delicate states are often extremely sensitive to crystalline disorder and impurities $[3,4]$. Hence, robust material science research is crucial to ensure that well-characterized and phase-pure samples are provided for advanced measurement techniques such as neutron scattering, angle-resolved photoemission spectroscopy, and scanning tunneling microscopy [5]. In this paper, we report a comprehensive powder synthesis and characterization study of the layered honeycomb quantum material $\mathrm{Na}_{2} \mathrm{RuO}_{3}$. We determine its ground state to be a correlated electron metal, which is at odds with previous claims of insulating antiferromagnetism [6].
Layered honeycomb structures have been identified as having the potential to host exotic ground states, a well-known example of which is the honeycomb iridates $A_{2} \operatorname{IrO}_{3}(A=$ $\mathrm{Na}, \mathrm{Li})$. In these $\mathrm{Ir}^{4+}\left(5 d^{5}\right)$ materials, the strong spin-orbit coupling (SOC) stabilizes a $J_{\text {eff }}=1 / 2$ Mott-insulating state. The combination of strong SOC, intrinsic geometric frustration, and other competing terms in the Hamiltonian favors highly anisotropic Kitaev exchange interactions [7,8]. Theoretical predictions suggest that the Kitaev physics associated with these interactions may lead to exotic phases such as topological insulators [9] and a quantum spin liquid state [10,11] (QSL). However, such novel ground states have not yet been realized in these systems largely due to competing isotropic interactions (Heisenberg) that stabilize magnetic order and/or lattice distortions that relieve geometrical frustration.

In this context, the study of the honeycomb ruthenate counterparts $\left(A_{2} \mathrm{RuO}_{3}\right.$, with $\left.A=\mathrm{Na}, \mathrm{Li}\right)$ provides a new path to explore systems with unusual physics emerging from the interplay of SOC and electronic correlations. These materials feature a $\mathrm{Ru}^{4+}\left(4 d^{4}\right)$ configuration, which in the presence of a strong octahedral crystal field, and intermediate strength 
SOC and Hund's couplings, yields a $t_{2 g}^{4}$ ground-state manifold with $J_{\text {eff }}=0$. For such systems it has recently been proposed that superexchange interactions lead to excitonic Van Vleck-like magnetism, and above a quantum critical point Bose-Einstein condensation of the higher-lying $J_{\text {eff }}=1$ triplet occurs [12-14]. The inherent bond-directionality of the $J_{\text {eff }}$ states renders the ground state exquisitely sensitive to the lattice connectivity. In the case of the honeycomb lattice, it is predicted that a novel type of spin liquid may be realized [15].

While interest in the honeycomb ruthenates has been largely concentrated in $\mathrm{Li}_{2} \mathrm{RuO}_{3}$ due to the presence of dimerization and the formation of an exotic valence bond liquid phase [16-18], studies of the physical properties of $\mathrm{Na}_{2} \mathrm{RuO}_{3}$ are somewhat scarce and unclear. Early studies by Mogare et al. [19] identified the crystal structure and synthesis conditions of $\mathrm{Na}_{2} \mathrm{RuO}_{3}$, noting that the material suffered from cation disorder within the honeycomb plane. More recently, Cao and co-workers [6] claimed to synthesize single crystals, determining $\mathrm{Na}_{2} \mathrm{RuO}_{3}$ to be an insulating antiferromagnet with a Néel temperature around $30 \mathrm{~K}$ from bulk susceptibility and heat-capacity measurements. Subsequently, Gapontsev et al. [20] used x-ray absorption spectroscopy, resistivity, and density functional theory calculations to propose a model of the magnetic structure in the ground state. In parallel, $\mathrm{Na}_{2} \mathrm{RuO}_{3}$ has been identified as a candidate material for a cathode for sodium batteries [21,22]. It has been demonstrated that it exhibits a remarkably high reversibility of electrochemical $\mathrm{Na}$ insertion/deinsertion combined with a high specific capacity [23]. From all these studies, $\mathrm{Na}_{2} \mathrm{RuO}_{3}$ has been identified to crystallize in one of the two existing polymorphs: a disordered- $\mathrm{Na}_{2} \mathrm{RuO}_{3}$ with space group $R \overline{3} m$ [23] and randomly distributed $\left[\mathrm{Na}_{1 / 3} \mathrm{Ru}_{2 / 3}\right] \mathrm{O}_{2}$ slabs, and an ordered- $\mathrm{Na}_{2} \mathrm{RuO}_{3}[6,19,21,22]$ with space group $C 2 / c$ (or $C 2 / m$ ) and honeycomb-ordered $\left[\mathrm{Na}_{1 / 3} \mathrm{Ru}_{2 / 3}\right] \mathrm{O}_{2}$ slabs (see Fig. 1 for the $C 2 / c$ crystal structure). Our interest is in the ordered phase, which might be expected to host exotic physics in the context of a $d^{4}$ spin-orbit coupled Mott insulator [15]. The purpose of the current study is to revisit the synthesis and characterization of low-temperature properties to clarify the ground state of the ordered phase of $\mathrm{Na}_{2} \mathrm{RuO}_{3}$. Remarkably, we find no evidence of magnetism in this material, identifying it instead as a paramagnetic, correlated electron metal. We discuss the possible reasons why this material was miscategorized.

\section{EXPERIMENT}

\section{A. Powder synthesis}

The synthesis of polycrystalline $\mathrm{Na}_{2} \mathrm{RuO}_{3}$ consisted of two steps, following similar procedures present in Refs. [19,24]. The first part involved the synthesis of $\mathrm{Na}_{2} \mathrm{RuO}_{4}$, prepared by solid-state reaction from stoichiometric amounts of $\mathrm{Na}_{2} \mathrm{O}_{2}$ and $\mathrm{RuO}_{2}$. The powders were ground and mixed in an agate mortar under argon. The mixtures were placed in an alumina crucible and heated to $450^{\circ} \mathrm{C}$ for $5 \mathrm{~h}, 530^{\circ} \mathrm{C}$ for $10 \mathrm{~h}$, and $630^{\circ} \mathrm{C}$ for $20 \mathrm{~h}$ under oxygen atmosphere with a temperature rate of $50{ }^{\circ} \mathrm{C} / \mathrm{h}$. The phase composition of the resulting powder was checked using a Rigaku Miniflex 600 x-ray powder diffractometer, with the resulting diffraction pattern showing

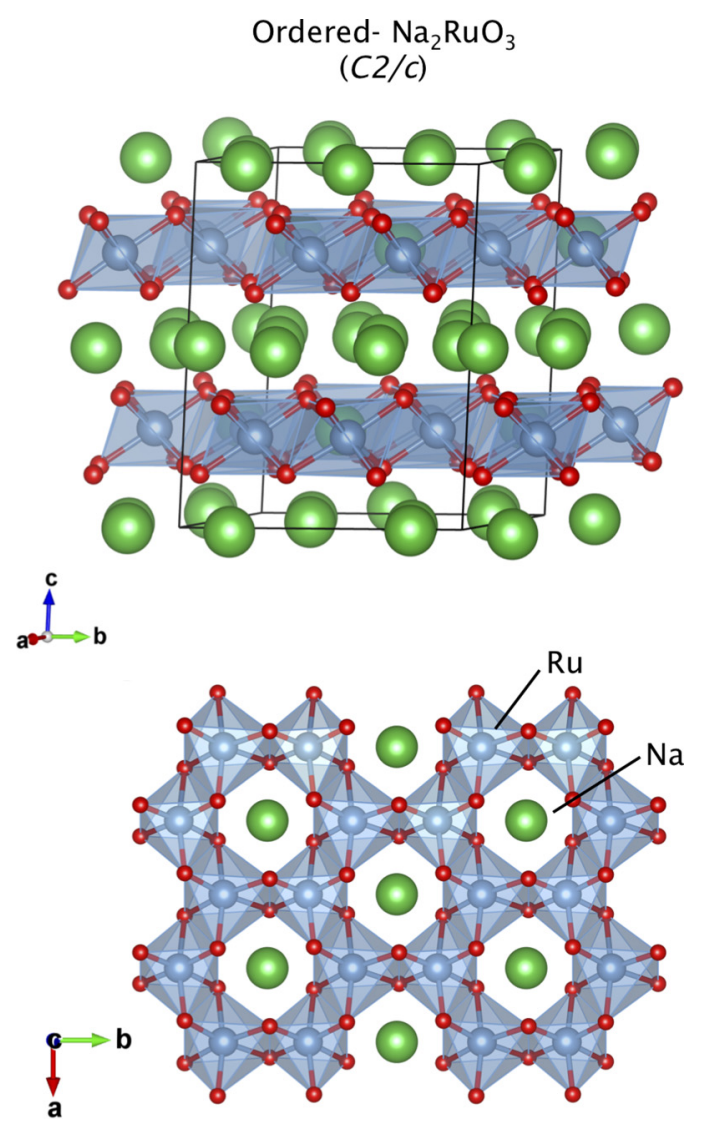

FIG. 1. Crystal structure of the ordered phase of $\mathrm{Na}_{2} \mathrm{RuO}_{3}$ in the $C 2 / c$ space group. Oxygen ions are drawn in red, $\mathrm{Na} / \mathrm{Ru}$ cations are showed in green/blue. Ordered $\mathrm{Na}_{2} \mathrm{RuO}_{3}$ has the honeycomb-type cation ordering in the $\left[\mathrm{Na}_{1 / 3} \mathrm{Ru}_{2 / 3}\right] \mathrm{O}_{2}$ slab. Stacking faults occur through the occasional shift of the $\left[\mathrm{Na}_{1 / 3} \mathrm{Ru}_{2 / 3}\right] \mathrm{O}_{2}$ layers perpendicular to the stacking direction, which in this case is the $c$-axis.

pure $\mathrm{Na}_{2} \mathrm{RuO}_{4}$ phase. The second step consisted of reducing the $\mathrm{Na}_{2} \mathrm{RuO}_{4}$ under argon flow at $950{ }^{\circ} \mathrm{C}$ for $24 \mathrm{~h}$ using a tube furnace. The resulting product was a black homogeneous $\mathrm{Na}_{2} \mathrm{RuO}_{3}$ powder, which was found to be mildly air-sensitive. Efforts to make single crystals via flux growth were unsuccessful, due in part to the volatility of the material and the requirement of having a reducing atmosphere to stabilize the $\mathrm{Ru}^{4+}$ ion.

Attempts to synthesize $\mathrm{Na}_{2} \mathrm{RuO}_{3}$ from $\mathrm{Na}_{2} \mathrm{RuO}_{4}$ using other synthesis temperatures and atmospheric conditions were also conducted and are displayed in the phase diagram of Fig. 2. The phase homogeneity of the different batches was verified by powder $x$-ray diffraction [25]. In general, the optimum conditions for the synthesis of the ordered $\mathrm{Na}_{2} \mathrm{RuO}_{3}$ phase was found to be in the region corresponding to synthesis temperatures between 850 and $1050^{\circ} \mathrm{C}$ and partial oxygen pressure in one bar varying from $0.001 \%(5 \mathrm{~N}$ argon atmosphere) to $0.1 \%$. In general, the final structure was sensitive to final synthesis conditions; for example, temperatures above $1050^{\circ} \mathrm{C}$ under an argon atmospheric condition produced samples with a high degree of in-plane Ru-Na disorder characterized by an $R \overline{3} m$ space group. On the other hand, for temperatures ranging from 850 to $1050^{\circ} \mathrm{C}$ under the same 


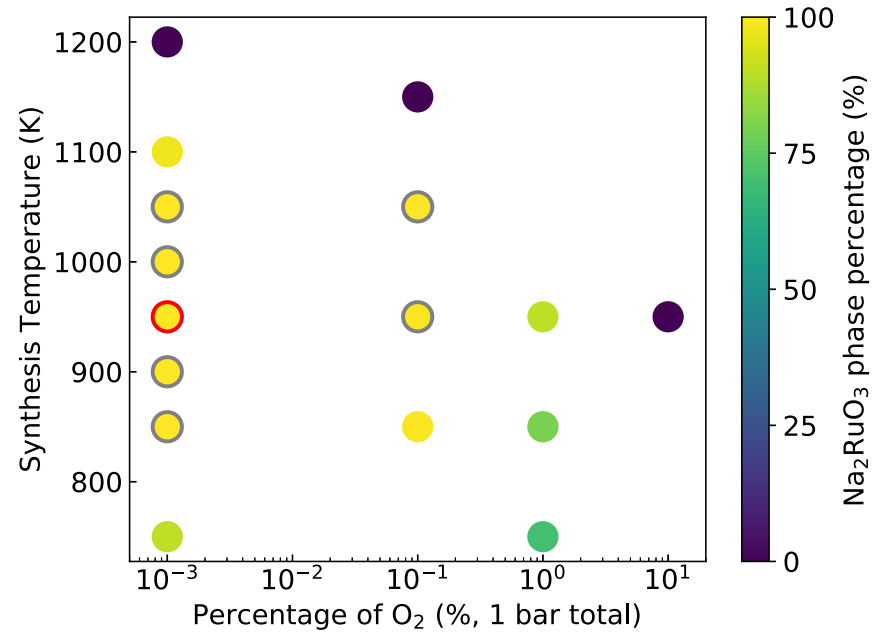

FIG. 2. Phase diagram of the synthesis conditions of $\mathrm{Na}_{2} \mathrm{RuO}_{3}$. The markers denote the conditions at which the synthesis attempts were conducted. The color scale represents the estimated percentage of the ordered $\mathrm{Na}_{2} \mathrm{RuO}_{3}$ phase compared to foreign phases obtained from the $\mathrm{x}$-ray diffraction measurements. The foreign phases include other Na-Ru-O compositions such as disordered $\mathrm{Na}_{2} \mathrm{RuO}_{3}$, $\mathrm{Na}_{2} \mathrm{RuO}_{4}, \mathrm{NaRuO}_{2}, \mathrm{Na}_{3} \mathrm{RuO}_{4}$, and $\mathrm{Na}_{27} \mathrm{Ru}_{14} \mathrm{O}_{48}$. The circles with a gray edge indicate the optimum synthesis conditions. The circle with a red edge highlights the synthesis conditions used to prepare the samples for all the measurements presented in this paper.

conditions, the crystal structure of the powders was mostly characterized by the ordered $C 2 / c$ space group [25]. A complete systematic study of the bulk properties of all batches was not performed and is beyond the scope of this study. Note that despite ranges of temperature and partial oxygen pressure having been identified as optimum conditions for the synthesis of ordered $\mathrm{Na}_{2} \mathrm{RuO}_{3}$ (see Fig. 2), all the samples used in the various experiments reported in this paper were prepared by heating $\mathrm{Na}_{2} \mathrm{RuO}_{4}$ under argon flow at $950{ }^{\circ} \mathrm{C}$ for $24 \mathrm{~h}$.

\section{B. Characterization methods}

The crystallographic structure of the ordered $\mathrm{Na}_{2} \mathrm{RuO}_{3}$ was verified by time-of-flight neutron powder diffraction (TOF-NPD) measurements on the WISH instrument at the ISIS spallation neutron source, UK. The powder $(\sim 3.5 \mathrm{~g}$ total mass) was finely ground and enclosed in a thin-walled cylindrical vanadium can. The obtained powder diffraction patterns collected for temperatures ranging from $T=100$ to $1.5 \mathrm{~K}$ using detector banks $2-5$ were analyzed by Rietveld refinement as implemented in the TOPAS software (TOPAS 6.0, Bruker AXS, 2017). For each diffraction pattern, Chebyshev polynomials of 24th order were used in order to model the complex corrugated background. Structural parameters, such as lattice parameters, atomic positions, occupancies, isotropic displacement parameters, and sample-dependent peak shape contributions, were constrained over the different banks. More details about the Rietveld refinement can be found in the Supplemental Material [25].

Bulk characterization of the powders consisted of magnetic susceptibility $(\chi)$ measurements in a Quantum Design superconducting quantum interference device (SQUID), heat capacity $\left(C_{p}\right)$, and four-probe resistance $(R)$ measurements in a Quantum Design physical properties measurement system (PPMS). The heat-capacity measurement was made on a sintered, cylindrical pellet with specific dimensions to adapt to the mounting on the PPMS addenda, i.e., large surface area and thin sample to enable good thermalization. The two-tau analysis method was employed, and a high coupling constant $(>90 \%)$ was measured at all temperatures demonstrating that the extracted time constant represents the relaxation of the sample with the external thermal reservoir. Furthermore, the relaxation curves were well-fitted to the two-tau model, and no distortion in the raw relaxation curves was observed. Energydispersive $\mathrm{x}$-ray spectroscopy (EDX) was used to investigate the $\mathrm{Na}: \mathrm{Ru}$ ratio on a representative batch of powders using a JEOL JSM-6610LV with Oxford Instruments EDX. To this end, the powders were pelletized and cleaved to expose fresh surfaces, and the measurements were conducted in six different areas of the sample. The measurements gave an average $\mathrm{Na}: \mathrm{Ru}$ ratio of $\sim 2.11$, suggesting that the samples are slightly $\mathrm{Ru}$-deficient. The deficiency of $\mathrm{Ru}$ is likely related to the loss of $\mathrm{RuO}_{2}$ during the reduction process under an argon atmosphere. While an excess of sodium might be present in our samples, due to the flexibility of the structure and as demonstrated by studies of battery material $[21,22]$, the high volatility of $\mathrm{Na}_{2} \mathrm{O}$ at $950{ }^{\circ} \mathrm{C}$ compared to $\mathrm{RuO}_{2}$ suggests that the excess of sodium in the structure of $\mathrm{Na}_{2} \mathrm{RuO}_{3}$ is unlikely to occur.

The photoelectron spectroscopy (PES) measurements reported here were performed with a frequency-converted continuous-wave laser and a hemispherical electron spectrometer. The photon energy of $6.01 \mathrm{eV}$ used in these experiments provides an increased bulk sensitivity as compared to the more widely used energies of 20-100 eV. The measurements were performed on pressed powders cleaved under ultrahigh vacuum, and the data were collected for temperatures varying between 300 and $10 \mathrm{~K}$.

The inelastic neutron scattering measurements were performed at the time-of-flight chopper spectrometer MARI of the ISIS Facility with incident neutron energies of 180 and $15 \mathrm{meV}$ at temperatures of 5,7 , and $300 \mathrm{~K}$. Powder of $\mathrm{Na}_{2} \mathrm{RuO}_{3}(\approx 10 \mathrm{~g})$ was wrapped in a thin Al-foil and mounted inside a thin-walled Al-can which was cooled down to $5 \mathrm{~K}$ inside a top-loading closed-cycle refrigerator (CCR) with Heexchange gas.

\section{RESULTS}

\section{A. Crystal structure}

The TOF-NPD patterns of $\mathrm{Na}_{2} \mathrm{RuO}_{3}$ were collected for temperatures ranging from $T=100 \mathrm{~K}$ (Fig. 3) to $1.5 \mathrm{~K}$ [25]. No extra peaks were observed in the entire temperature range measured, indicating no change in crystal symmetry or the appearance of magnetic peaks. No secondary phases were detected. The TOF-NPD patterns were refined by the Rietveld method using existing models for $\mathrm{Na}_{2} \mathrm{RuO}_{3}$ present in the literature $[19,21,22]$. While the disordered crystal structure model (space group $R \overline{3} m$ ) fitted most of the intense reflections, several peaks were not covered by this space group, 


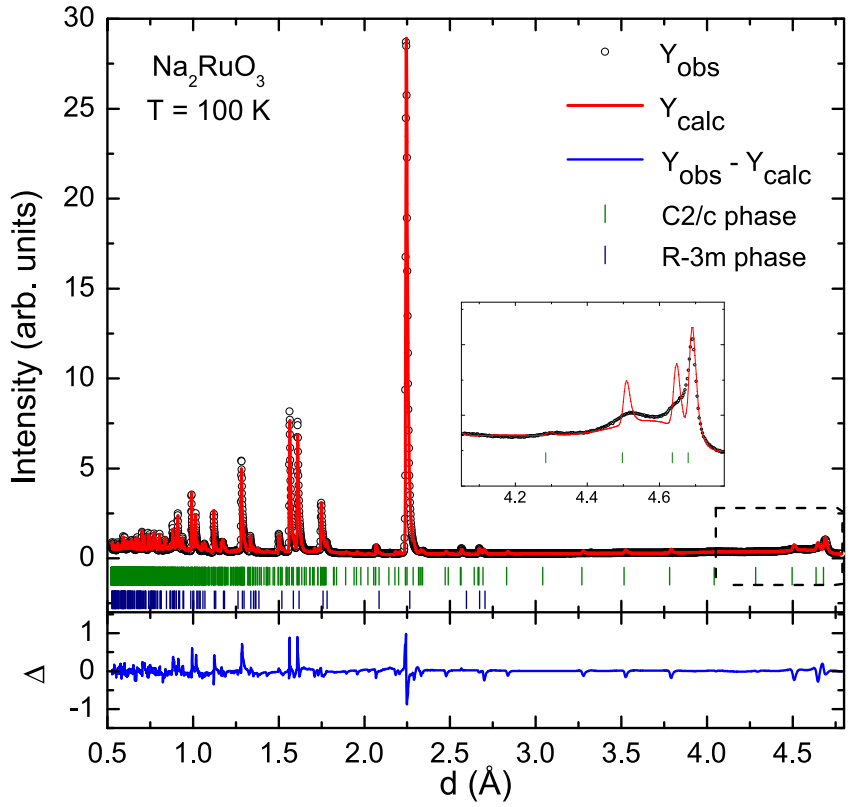

FIG. 3. Powder neutron diffraction obtained from the WISH instrument at ISIS at $100 \mathrm{~K}$ using bank 5. Black open circles indicate the experimental data points, and the red solid line is the Rietveld refinement. The Bragg peak positions of the monoclinic and rhombohedral phases are indicated by the green and dark blue vertical ticks, respectively. The blue line shows the difference between the data and the simulated curve. Details of the refinement in the other detector banks can be found in the Supplemental Material [25].

especially in the region of the inset of Fig. 3. The presence of these reflections demonstrates that the majority phase is the ordered $C 2 / c$ structure. These reflections also exhibit a characteristic triangular shape profile, which is a signature of stacking fault disorder. The intrinsic stacking fault behavior arises from deviations of the theoretical optimal alternating stacking sequence of a $\mathrm{Na}$ layer followed by a $\mathrm{NaRu}_{2}$ layer. To cover all the reflections present in the neutron powder pattern, we have used a two-phase model where the ordered $C 2 / c$ and disordered $R \overline{3} m$ phases were refined simultaneously. Figure 3 shows the Rietveld refinement of the $\mathrm{Na}_{2} \mathrm{RuO}_{3}$ powder neutron diffraction pattern at $T=100 \mathrm{~K}$ using this approach. The refined parameters and atomic positions can be found in Tables I and II. Details of the refinement at $T=1.5 \mathrm{~K}$ can be found in the Supplemental Material [25].

The two-phase model was also used by Mogare et al. [19] and resulted in a significant volume contribution of the disordered phase in their x-ray powder diffraction refinement, indicating a high degree of disorder in the structure of their material. In contrast, our Rietveld refinement shows a weight percentage of the ordered $C 2 / c$ phase of $93 \%$. While the twophase model can predict most of the reflections present in the inset of Fig. 3, it is still not enough to account for the underlying stacking-fault behavior in $\mathrm{Na}_{2} \mathrm{RuO}_{3}$, which is discussed in more detail in Sec. IV. Moreover, the model overestimates the crystallinity of the material and creates some additional phase peaks (such as in the region $d \approx 2.5-4 \AA$ ) that cannot be found in the observed data.
TABLE I. Final parameters of the Rietveld refinement of $\mathrm{Na}_{2} \mathrm{RuO}_{3}$ using the two-phase model at $T=100 \mathrm{~K}$. Residual factors and goodness of fit $(\mathrm{GoF})$ are the overall factors for all detector banks and are defined as in the TOPAS software. The low GoF factor is explained by an overestimated $R_{\exp }$ factor. The site occupancies were kept fixed throughout the refinement.

\begin{tabular}{|c|c|c|}
\hline Formula (nominal) & \multicolumn{2}{|c|}{$\mathrm{Na}_{2} \mathrm{RuO}_{3}$} \\
\hline Crystal system & monoclinic & rhombohedral \\
\hline Space group & $C 2 / c$ (No. 15$)$ & $R \overline{3} m$ (No. 166) \\
\hline \multicolumn{3}{|l|}{ Lattice parameters } \\
\hline$a(\AA)$ & $5.4131(1)$ & $3.1689(9)$ \\
\hline$b(\AA)$ & $9.3590(2)$ & \\
\hline$c(\AA)$ & $10.8113(1)$ & $16.0372(55)$ \\
\hline$\beta(\operatorname{deg})$ & $99.642(2)$ & \\
\hline Unit-cell volume $\left(\AA^{3}\right)$ & $539.977(21)$ & $139.466(94)$ \\
\hline Unit-cell mass (u) & 1560.382 & 390.095 \\
\hline Formula units & $Z=8$ & $Z=3$ \\
\hline Weight percentage & $93.935(4) \%$ & $6.065(4) \%$ \\
\hline$R_{\exp }$ & \multicolumn{2}{|c|}{70.508} \\
\hline$R_{\exp }^{\prime}$ & \multicolumn{2}{|c|}{75.024} \\
\hline$R_{\mathrm{wp}}$ & \multicolumn{2}{|c|}{6.885} \\
\hline$R_{\mathrm{wp}}^{\prime}$ & \multicolumn{2}{|c|}{7.326} \\
\hline$R_{\mathrm{p}}$ & \multicolumn{2}{|c|}{9.291} \\
\hline$R_{\mathrm{p}}^{\prime}$ & \multicolumn{2}{|c|}{17.820} \\
\hline $\mathrm{GoF}$ & \multicolumn{2}{|c|}{0.098} \\
\hline
\end{tabular}

\section{B. Bulk properties}

Figure 4(a) shows the temperature-dependent magnetic response $\chi(T)$ of $\mathrm{Na}_{2} \mathrm{RuO}_{3}$ in an applied magnetic field of $1 \mathrm{~T}$. The data were collected from field-cooled powders with a total mass of $0.3 \mathrm{~g}$. No evidence of magnetic ordering is observed in $\chi(T)$ down to $2 \mathrm{~K}$, in contrast with a previous report on single crystals of $\mathrm{Na}_{2} \mathrm{RuO}_{3}$ where a sharp antiferromagnetic transition was observed at $T_{N}=30 \mathrm{~K}$ [6]. No appreciable difference is observed between data collected under zerofield or field-cooled conditions. Above $50 \mathrm{~K}$, the magnetic susceptibility is weakly temperature-dependent and is likely dominated by the Pauli term. To estimate the intrinsic susceptibility, $\chi_{0}$, we first fit the paramagnetic upturn below $50 \mathrm{~K}$ to a Curie-Weiss law and subtract it from the raw data. Next, we subtract the isotropic core diamagnetism [26] $\left(\chi_{D} \approx-5.02 \times\right.$ $\left.10^{-5} \mathrm{emu} / \mathrm{mol}\right)$. This gives an intrinsic $\chi_{0} \sim 1.42(2) \times 10^{-3}$ $\mathrm{emu} / \mathrm{mol}$ Oe. The low-temperature Curie-Weiss contribution is equivalent to approximately $10 \%$ by mass of the $S=1$ impurity, the origin of which we discuss later.

Specific-heat data show no sign of a phase transition down to $1.9 \mathrm{~K}$, as displayed in Fig. 4(b). Fitting the lowtemperature region $(1.9 \leqslant T \leqslant 12 \mathrm{~K})$ to $C / T=\gamma+\beta T^{2}$ yields an electronic coefficient of $\gamma=11.7(2) \mathrm{mJ} / \mathrm{Ru} \mathrm{mol}$ $\mathrm{K}^{2}$ and a phononic contribution of $\beta=0.198(2) \mathrm{mJ} / \mathrm{Ru}$ mol K${ }^{4}$ [inset of Fig. 4(b)]. The electronic coefficient is comparable to the values found for $\mathrm{Sr}_{2} \mathrm{RhO}_{4}$ [27] and Ladoped $\left(\mathrm{Sr}_{1-x} \mathrm{La}_{x}\right)_{3} \mathrm{Ir}_{2} \mathrm{O}_{7}[28,29](x \geqslant 0.05)$ and suggests that $\mathrm{Na}_{2} \mathrm{RuO}_{3}$ may be a moderately correlated metal. The experimental values of $\chi_{0}$ and $\gamma$ give a Wilson ratio of $R_{W} \approx 8.9(1)$, which is much higher than the unity value for a free-electron gas. The enhanced value of $R_{W}$ suggests that $\mathrm{Na}_{2} \mathrm{RuO}_{3}$ is near an instability [30] and that it might be regarded as a moderately correlated metal with enhanced spin susceptibility. 
TABLE II. Final refined atomic site parameters of the Rietveld refinement of $\mathrm{Na}_{2} \mathrm{RuO}_{3}$ at $T=100 \mathrm{~K}$ using the two-phase model.

\begin{tabular}{|c|c|c|c|c|c|}
\hline Atom & $x$ & $y$ & $z$ & Occupancy & $B_{\text {iso }}$ \\
\hline \multicolumn{6}{|c|}{ monoclinic phase } \\
\hline $\mathrm{Na} 1$ & $0.2367(13)$ & $0.5956(7)$ & $0.0050(8)$ & 1 & $0.71(5)$ \\
\hline $\mathrm{Na} 2$ & 0.25 & 0.25 & 0 & 1 & \\
\hline $\mathrm{Na} 3$ & 0 & $0.8958(12)$ & 0.25 & 1 & \\
\hline $\mathrm{Ru} 1$ & 0 & $0.2654(7)$ & 0.25 & 1 & $0.49(4)$ \\
\hline $\mathrm{Ru} 2$ & 0 & $0.5975(7)$ & 0.25 & 1 & \\
\hline $\mathrm{O} 1$ & $0.1295(9)$ & $0.0763(6)$ & $0.1495(5)$ & 1 & $1.62(3)$ \\
\hline $\mathrm{O} 2$ & $0.1497(9)$ & $0.4072(6)$ & $0.1470(5)$ & 1 & \\
\hline $\mathrm{O} 3$ & $0.1554(10)$ & $0.7497(8)$ & $0.1479(6)$ & 1 & \\
\hline \multicolumn{6}{|c|}{ rhombohedral phase } \\
\hline $\mathrm{Na1}$ & 0 & 0 & 0 & 1 & $0.71(5)$ \\
\hline $\mathrm{Na} 2$ & 0 & 0 & 0.5 & $1 / 3$ & $0.49(4)$ \\
\hline $\mathrm{Ru}$ & 0 & 0 & 0.5 & $2 / 3$ & \\
\hline $\mathrm{O}$ & 0 & 0 & $0.2240(9)$ & 1 & $1.62(3)$ \\
\hline
\end{tabular}

No signatures of magnetic excitations of $\mathrm{Na}_{2} \mathrm{RuO}_{3}$ were found in the inelastic neutron scattering measurements. The INS powder spectra collected at $T=7$ and $5 \mathrm{~K}$ with two different incident neutron energies, 15 and $180 \mathrm{meV}$, respectively, are shown in Figs. 5(a) and 5(b). The color scale represents the observed intensity as a function of energy transfer and momentum transfer $(|Q|)$. We note an absence of a continuum of spin excitations at low $|Q|$ and low-energy transfer [25]. This behavior, characteristic of magnetic frustration, has been the most widely accepted evidence for a QSL state. The buildup of weak intensity at the high- $|Q|$ region in Fig. 5(b) comes from the phonon modes that become stronger at higher incident energies.

All the measurements mentioned above are consistent with our results from PES experiments. The inset of Fig. 4(a) shows the Fermi edges of polycrystalline $\mathrm{Na}_{2} \mathrm{RuO}_{3}$ at $T \leqslant 50 \mathrm{~K}$ with a photon energy of $6 \mathrm{eV}$. The PES measurements reveal a jump in the photoelectron intensity at the chemical potential, which is indicative of a small but finite density of states at the Fermi energy for all temperatures probed in these experiments. Similar PES experiments with a higher photon energy and correspondingly increased surface sensitivity did not detect any Fermi step. This strongly suggests that the PES data shown in the inset of Fig. 4(a) represent a finite bulk density of states at the chemical potential, consistent with the specific-heat measurements. Although not performed in this present study, band-structure calculations would be highly desirable to support the metallic nature of $\mathrm{Na}_{2} \mathrm{RuO}_{3}$ and to confirm existing calculations available in the materials project database [31].
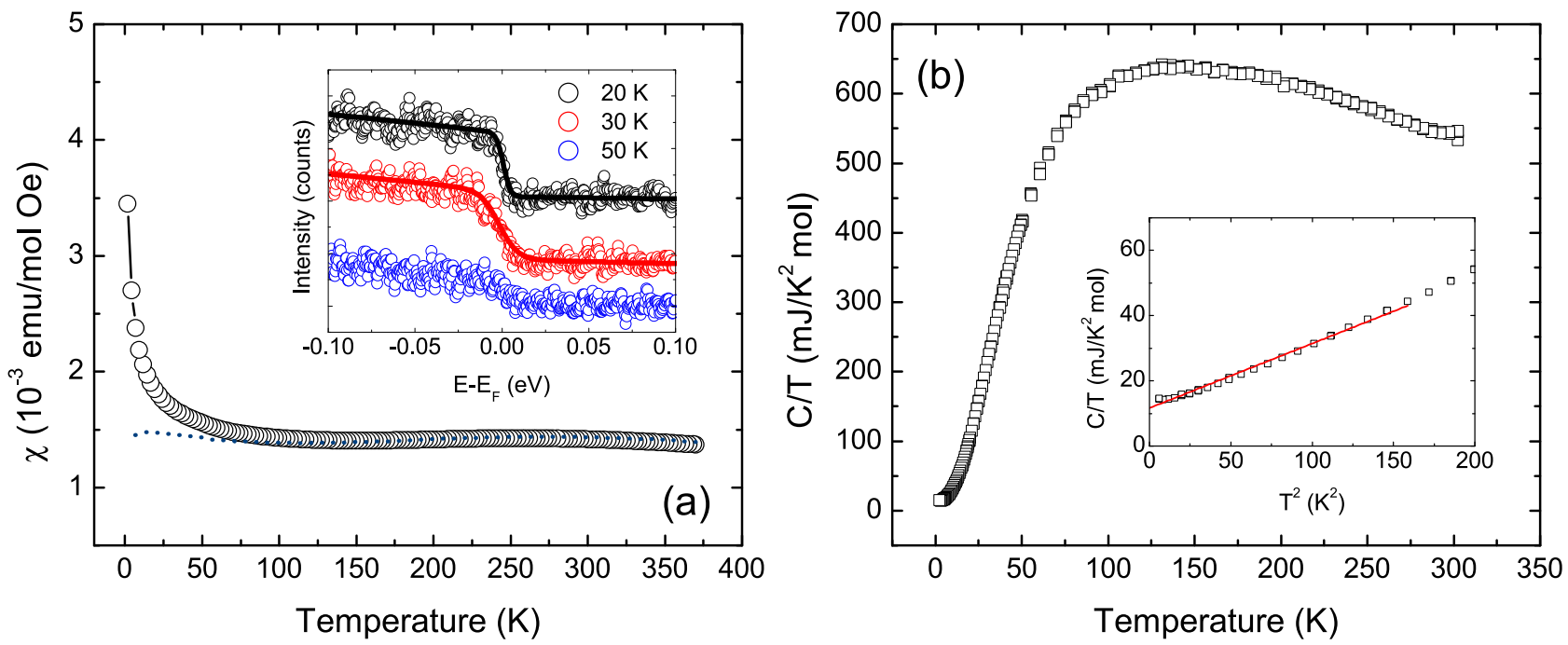

FIG. 4. (a) Magnetic susceptibility of polycrystalline $\mathrm{Na}_{2} \mathrm{RuO}_{3}$ for an applied field of $1 \mathrm{~T}$. The raw data (circles and solid line) are shown along with the presumed intrinsic $\chi_{0}$ (dotted line) after subtracting a low-temperature Curie-like $1 / T$ contribution. The inset shows the Fermi edge of $\mathrm{Na}_{2} \mathrm{RuO}_{3}$ polycrystalline pellets at different temperatures revealed by PES measurements (open circles). The photoemission data were fitted to a Fermi-Dirac function multiplied by a polynomial background and convoluted with a Gaussian. The Gaussian function has a FWHM of $5 \mathrm{meV}$, which is the expected resolution of the experiment. The data were stacked vertically for better visualization of the fitting results, which are showed by solid lines. We have not fitted the data at $50 \mathrm{~K}$ due to their low signal-to-noise ratio. The fitting results from the 20 and $30 \mathrm{~K}$ data revealed that a gap can be excluded within our experimental accuracy. (b) Specific-heat data as a function of temperature. The inset shows a linear fit (solid line) in a $C / T$ vs $T^{2}$ plot. 

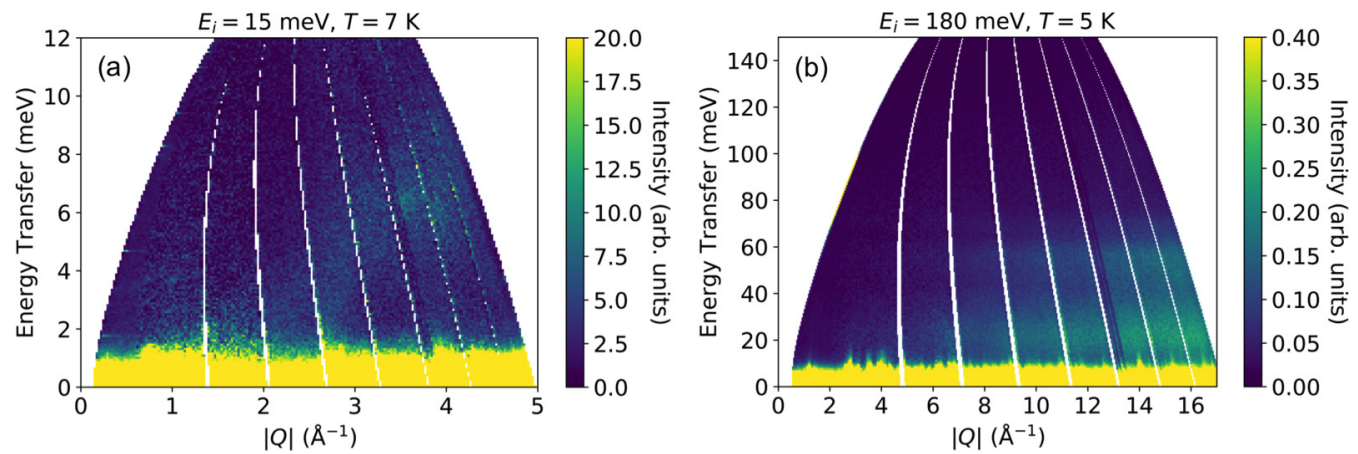

FIG. 5. INS spectra of $\mathrm{Na}_{2} \mathrm{RuO}_{3}$ at low temperatures obtained using incident energies of (a) $15 \mathrm{meV}$ and (b) $180 \mathrm{meV}$. No magnetic excitations are observed at any incident energy of the neutrons.

In contrast to all of our other data, the resistance was found to increase substantially on cooling, indicating an insulating behavior, as observed in Fig. 6. These measurements were carried out using a conventional four-probe terminal method on a cold-pressed pellet, which was sintered in $\arg$ on at $850^{\circ} \mathrm{C}$ for $1 \mathrm{~h}$ to reduce the effects of grain boundaries. The annealing protocol did not degrade the sample, as confirmed by the x-ray diffraction measurements on the resulting pellets.

\section{DISCUSSION}

The structural and chemical characterization of our powders demonstrates that we have synthesized a nearly phase-pure compound of ordered $\mathrm{Na}_{2} \mathrm{RuO}_{3}$. However, a complete solution of the crystal structure is hindered by the presence of broad diffraction peaks, which are common for $A_{2} M_{3} \quad(A=\mathrm{Li}, \mathrm{Na}$ and $M=\mathrm{Ir}, \mathrm{Ru})$ with honeycomb ordered layers, and it can be attributed to stacking disorder. Since in these layered materials the crystal grows perpendicular to the layer plane (here, along the $c$-axis), the ordered honeycomb layers can nucleate in different stacking position, creating stacking faults. The deviation from the ideal sequence of honeycomb stacked layers leads to the peculiar

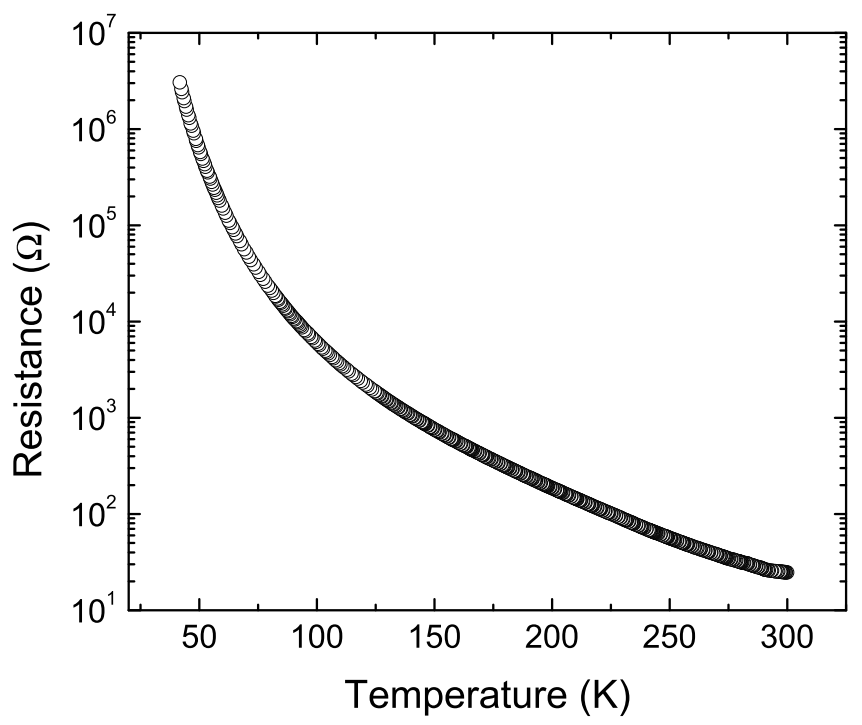

FIG. 6. Electrical resistance of polycrystalline $\mathrm{Na}_{2} \mathrm{RuO}_{3}$ as a function of temperature. triangular Warren-type peak shapes observed in Fig. 3. While stacking faults can be modeled by Rietveld refinement using combined x-ray diffraction datasets [32-35], our attempts to tackle this problem proved unsuccessful mainly due to the complex background and intrinsic asymmetry of the peak shapes present in the TOF-NPD data. Nevertheless, our TOFNPD analysis using the two-phase model reveals a high degree of ordering in the structure of our powders and suggests that the $C 2 / c$ crystal structure is a good candidate for the microstructural modeling of the stacking faults in $\mathrm{Na}_{2} \mathrm{RuO}_{3}$. Indeed, recent study on sodium-based batteries [22] shows that modeling of stacking faults using a similar space group $(C 2 / m)$ is possible when using combined x-ray diffraction data. Finally, the similarity of the phase percentage of the disordered phase $(\approx 6 \%)$ and the Curie-Weiss contribution to susceptibility at low temperature $(\approx 10 \%)$ leads us to suggest that the disordered phase might be responsible for the magnetic impurities. This is not unreasonable if the intraplane $\mathrm{Ru}$ disorder causes the electrons to localize and contribute $S=1$ moment per $\mathrm{Ru}^{+4}$ ion. The small amount of magnetic impurities may also be responsible for the enhanced Wilson ratio and can explain the absence of magnetic excitations in the INS data due to the proximity of a Stoner transition.

Interestingly, our results reveal that $\mathrm{Na}_{2} \mathrm{RuO}_{3}$ has a distinct ground state to that reported by Wang et al. [6]. Single crystals of $\mathrm{Na}_{2} \mathrm{RuO}_{3}$ were claimed to order antiferromagnetically below $T_{N}=30 \mathrm{~K}$ and to present a highly insulating ground state. We observe no evidence of magnetic order in our polycrystalline $\mathrm{Na}_{2} \mathrm{RuO}_{3}$ in any bulk or spectroscopic probe: neither the NPD nor bulk susceptibility show any feature at $30 \mathrm{~K}$. Coincidentally, the features in the susceptibility from Ref. [6] are remarkably similar to those observed in $\mathrm{Na}_{3} \mathrm{RuO}_{4}$, the structure of which consists of isolated tetramers of $\mathrm{Ru}^{5+}$ ions $(S=3 / 2)$ in a so-called lozenge configuration [36,37]. We suggest that the single crystals from their study have been miscategorized as $\mathrm{Na}_{2} \mathrm{RuO}_{3}$. Remarkably, the structure of $\mathrm{Na}_{2} \mathrm{RuO}_{3}$ is quite different from the isoelectronic sister compound $\mathrm{Li}_{2} \mathrm{RuO}_{3}$. In $\mathrm{Li}_{2} \mathrm{RuO}_{3}$, the polycrystalline lowtemperature phase adopts a strongly distorted honeycomb lattice and dimerization, leading to the formation of a singlet ground state [16-18], which is in contrast to $\mathrm{Na}_{2} \mathrm{RuO}_{3}$, suggesting that the small ionic radius of lithium plays a crucial role in stabilizing the singlet ground state.

The ground state of insulating ruthenates has been under debate in recent years due to the similarity of SOC, 
Hund's rule, and crystal-field energy scales. Traditionally, perovskite ruthenates have been understood by arguing that the octahedral crystal field lifts the degeneracy of the $d$ orbital manifold, and the four $4 d$ electrons fill the $t_{2 g}$ energy states to generate a low-spin $S=1$ moment. However, if one considers the effect of the moderate SOC of the $4 d$ electrons, the $t_{2 g}$ manifold could behave as a pseudo- $L_{\text {eff }}=1$ (or $p$-state) generating a $J_{\text {eff }}=0$ ground state with an excited state $J_{\text {eff }}=1$ at an energy $\lambda$ (SOC energy scale) above the ground state, as postulated in $\mathrm{Ca}_{2} \mathrm{RuO}_{4}[12,38]$. Hence, one might expect a Van Vleck behavior in the magnetism, where the mixing of the ground state and excited levels produces a temperature-independent contribution to the susceptibility that might explain the susceptibility of $\mathrm{Na}_{2} \mathrm{RuO}_{3}$. However, we believe that there are reasons to rule out this scenario in $\mathrm{Na}_{2} \mathrm{RuO}_{3}$. First, the energy scale of the SOC in ruthenates is between 50 and $100 \mathrm{meV}$ [39], yet we do not observe any excited states in the INS data below $150 \mathrm{meV}$, although we cannot rule out magnetic excitations at higher energy, which could be probed by resonant inelastic x-ray scattering. Secondly, a $J_{\text {eff }}=0$ state with unquenched orbital angular momentum requires a high-symmetry octahedral environment around the $\mathrm{Ru}^{4+}$ ion, but the $\mathrm{Na}_{2} \mathrm{RuO}_{3}$ has a large octahedra distortion of $7 \%\left[r_{l} / r_{s}\right.$, where $r_{l}\left(r_{s}\right)$ is the longest (shortest) $\mathrm{Ru}-\mathrm{O}$ bond], much greater than the Jahn-Teller distortions observed in $\mathrm{Ca}_{2} \mathrm{RuO}_{4}(\approx 1.5 \%$ at $10 \mathrm{~K})$ [40]. Such a large distortion is far more likely to quench the orbital magnetism within the $t_{2 g}$ manifold and stabilize a configuration with a filled $d_{x y}$ orbital and half-filled $d_{y z, z x}$ orbitals, leading to an $S=1$ state in the localized limit.

Finally, the discrepancy between the bulk measurements in our polycrystalline $\mathrm{Na}_{2} \mathrm{RuO}_{3}$ is perhaps most intriguing. Taken together, the susceptibility, heat capacity, and photoemission measurements indicate that this material has a Fermi-liquid-like ground state. However, the insulating behavior in the resistance measurements appears to contradict this hypothesis. We believe that the source of the discrepancy is the transport measurements made on our powder samples. It is a common practice in the community to determine the resistivity of powdered materials using cold or pressed samples and four-terminal dc voltage measurements. However, this can often lead to misleading results; resistance measurements on powdered samples contain contributions from two sources: intrinsic and grain boundary (extrinsic), and it is challenging to separate them. The electron hopping between grains can be modeled as a large energy barrier through which the electrons must tunnel, similar to electron motion in a scanning tunneling microscope. This intergrain hopping is the origin of the activation-like exponential increase in the resistance as the temperature is lowered and usually dominates over the intrinsic resistance of a grain. Moreover, the grain boundaries will introduce capacitance into the measurement circuit, thus ideally the complex impedance should be measured as a function of frequency and temperature so that the real part of the intrinsic impedance can be extracted. For a good example see
Ref. [41], where the extracted grain boundary resistance in a pressed pellet of $\mathrm{UO}_{2}$ had an activation-like temperature dependence with a barrier energy of $0.13 \mathrm{eV}$ and the grain boundary resistance was an order of magnitude larger than the observed intrinsic resistance. It is also worth pointing out that for transport measurements on powders, reproducibility is generally poor; each pressed pellet will have a unique grain boundary resistance due to a large number of factors, including porosity, grain stoichiometry, hygroscopicity, and air sensitivity. In general, single crystals are the most reliable form on which to measure transport properties, and measurements on powders should be treated with caution. For our system, if we neglect the resistivity dataset, the simple conclusion is that $\mathrm{Na}_{2} \mathrm{RuO}_{3}$ is a moderately correlated electron metal with modest magnetic enhancements, a conclusion supported by photoemission spectroscopy experiments.

\section{CONCLUDING REMARKS}

We have performed a detailed study of the synthesis and characterisation of polycrystalline $\mathrm{Na}_{2} \mathrm{RuO}_{3}$ by using neutron diffraction, inelastic neutron scattering as well as susceptibility, heat capacity and photemission measurements. No sign of magnetic ordering or magnetic frustration is observed down to $1.5 \mathrm{~K}$. Both magnetic susceptibility and heat capacity data indicate characteristics of significant electron correlation, with a large temperature independent Pauli paramagnetism and moderate Sommerfeld coefficient. This is consistent with the observation of a Fermi edge in the PES measurements. In contrast, resistivity measurements show nonmetallic behavior, which may be attributed to grain boundary effects. Thus, we classify $\mathrm{Na}_{2} \mathrm{RuO}_{3}$ as a moderately correlated electron metal.

\section{ACKNOWLEDGMENTS}

We would like to thank G. Stenning and D. Nye for help with the instruments in the Materials Characterisation Laboratory at the ISIS Neutron and Muon Source. We also thank P. Manuel for help with the TOF-NPD measurements at WISH instrument and for useful comments and suggestions on our manuscript. Experiments at MARI instrument were supported by a beamtime allocation from the Science and Technology Facilities Council (STFC) under proposal RB1820455. We also acknowledge the use of the JEOL JSM-6610LV with Oxford Instruments EDX present in the Research Complex at Harwell $(\mathrm{RCaH})$. This research was supported by the UK Engineering and Physical Sciences Research Council (EPSRC) (Grants No. EP/N027671/1 and No. EP/N034694/1). C.D.D. was supported by the EPSRC Centre for Doctoral Training in the Advanced Characterisation of Materials under Grant No. EP/L015277/1. F.B. and E.C. were supported by the Swiss National Science Foundation (SNSF). H.J. has received funding from the European Union's Horizon 2020 research and innovation programme under the Marie Skłodowska-Curie Grant Agreement No. 701647.
[1] B. Keimer and J. E. Moore, Nat. Phys. 13, 1045 (2017).

[2] L. Savary and L. Balents, Rep. Prog. Phys. 80, 016502 (2016).

[3] L. Balents, Nature (London) 464, 199 (2010).
[4] D. N. Basov, R. D. Averitt, and D. Hsieh, Nat. Mater. 16, 1077 (2017).

[5] N. Samarth, Nat. Mater. 16, 1068 (2017). 
[6] J. C. Wang, J. Terzic, T. F. Qi, F. Ye, S. J. Yuan, S. Aswartham, S. V. Streltsov, D. I. Khomskii, R. K. Kaul, and G. Cao, Phys. Rev. B 90, 161110(R) (2014).

[7] G. Jackeli and G. Khaliullin, Phys. Rev. Lett. 102, 017205 (2009).

[8] J. Chaloupka, G. Jackeli, and G. Khaliullin, Phys. Rev. Lett. 105, 027204 (2010).

[9] Y. Chen, Y.-M. Lu, and H.-Y. Kee, Nat. Commun. 6, 6593 (2015).

[10] A. Kitaev, Ann. Phys. 321, 2 (2006).

[11] W. Witczak-Krempa, G. Chen, Y. B. Kim, and L. Balents, Annu. Rev. Condens. Matter Phys. 5, 57 (2014).

[12] G. Khaliullin, Phys. Rev. Lett. 111, 197201 (2013).

[13] O. N. Meetei, W. S. Cole, M. Randeria, and N. Trivedi, Phys. Rev. B 91, 054412 (2015).

[14] C. Svoboda, M. Randeria, and N. Trivedi, Phys. Rev. B 95, 014409 (2017).

[15] J. Chaloupka and G. Khaliullin, Phys. Rev. B 100, 224413 (2019).

[16] Y. Miura, Y. Yasui, M. Sato, N. Igawa, and K. Kakurai, J. Phys. Soc. Jpn. 76, 033705 (2007).

[17] S. A. J. Kimber, I. I. Mazin, J. Shen, H. O. Jeschke, S. V. Streltsov, D. N. Argyriou, R. Valentí, and D. I. Khomskii, Phys. Rev. B 89, 081408(R) (2014).

[18] J. Park, T.-Y. Tan, D. T. Adroja, A. Daoud-Aladine, S. Choi, D.-Y. Cho, S.-H. Lee, J. Kim, H. Sim, T. Morioka, H. Nojiri, V. V. Krishnamurthy, P. Manuel, M. R. Lees, S. V. Streltsov, D. I. Khomskii, and J.-G. Park, Sci. Rep. 6, 25238 (2016).

[19] K. M. Mogare, K. Friese, W. Klein, and M. Jansen, Z. Anorg. Allg. Chem. 630, 547 (2004).

[20] V. V. Gapontsev, E. Z. Kurmaev, C. I. Sathish, S. Yun, J.-G. Park, and S. V. Streltsov, J. Phys.: Condens. Matter 29, 405804 (2017).

[21] B. Mortemard de Boisse, G. Liu, J. Ma, S.-i. Nishimura, S.C. Chung, H. Kiuchi, Y. Harada, J. Kikkawa, Y. Kobayashi, M. Okubo, and A. Yamada, Nat. Commun. 7, 11397 (2016).

[22] B. Mortemard de Boisse, M. Reynaud, J. Ma, J. Kikkawa, S.-i. Nishimura, M. Casas-Cabanas, C. Delmas, M. Okubo, and A. Yamada, Nat. Commun. 10, 2185 (2019).

[23] M. Tamaru, X. Wang, M. Okubo, and A. Yamada, Electrochem. Commun. 33, 23 (2013).

[24] M. Shikano, R. K. Kremer, M. Ahrens, H. J. Koo, M. H. Whangbo, and J. Darriet, Inorg. Chem. 43, 5 (2004).
[25] See Supplemental Material at http://link.aps.org/supplemental/ 10.1103/PhysRevMaterials.4.094202 for further information.

[26] G. A. Bain and J. F. Berry, J. Chem. Educ. 85, 532 (2008).

[27] R. S. Perry, F. Baumberger, L. Balicas, N. Kikugawa, N. J. C. Ingle, A. Rost, J. F. Mercure, Y. Maeno, Z. X. Shen, and A. P. Mackenzie, New J. Phys. 8, 175 (2006).

[28] T. Hogan, Z. Yamani, D. Walkup, X. Chen, R. Dally, T. Z. Ward, M. P. M. Dean, J. Hill, Z. Islam, V. Madhavan, and S. D. Wilson, Phys. Rev. Lett. 114, 257203 (2015).

[29] E. C. Hunter, An exploration of novel correlated electronic states in 5d transition metal oxides, Ph.D. thesis, University of Edinburgh, 2015.

[30] S.-I. Ikeda, Y. Maeno, S. Nakatsuji, M. Kosaka, and Y. Uwatoko, Phys. Rev. B 62, R6089 (2000).

[31] A. Jain, S. P. Ong, G. Hautier, W. Chen, W. D. Richards, S Dacek, S. Cholia, D. Gunter, D. Skinner, G. Ceder, and K. A. Persson, APL Materials 1, 011002 (2013).

[32] S. Bette, T. Takayama, K. Kitagawa, R. Takano, H. Takagi, and R. E. Dinnebier, Dalton Trans. 46, 15216 (2017).

[33] S. Bette, T. Takayama, V. Duppel, A. Poulain, H. Takagi, and R. E. Dinnebier, Dalton Trans. 48, 9250 (2019).

[34] S. Bette, B. Hinrichsen, D. Pfister, and E. Dinnebier, J. Appl. Crystallogr. 53, 76 (2020).

[35] L. Diehl, S. Bette, F. Pielnhofer, S. Betzler, I. Moudrakovski, G. A. Ozin, R. Dinnebier, and B. V. Lotsch, Chem. Mater. 30, 8932 (2018).

[36] J. T. Haraldsen, M. B. Stone, M. D. Lumsden, T. Barnes, R Jin, J. W. Taylor, and F. Fernandez-Alonso, J. Phys.: Condens. Matter 21, 506003 (2009).

[37] K. Regan, Q. Huang, and R. Cava, J. Solid State Chem. 178, 2104 (2005).

[38] A. Abragam and B. Bleaney, Electron Paramagnetic Resonance of Transition Ions (Clarendon, Oxford, 1970).

[39] A. Tamai, M. Zingl, E. Rozbicki, E. Cappelli, S. Riccò, A. de la Torre, S. McKeown Walker, F. Y. Bruno, P. D. C. King, W. Meevasana, M. Shi, M. Radović, N. C. Plumb, A. S. Gibbs, A. P. Mackenzie, C. Berthod, H. U. R. Strand, M. Kim, A. Georges, and F. Baumberger, Phys. Rev. X 9, 021048 (2019).

[40] D. Pincini, S. Boseggia, R. Perry, M. J. Gutmann, S. Riccò, L. S. I. Veiga, C. D. Dashwood, S. P. Collins, G. Nisbet, A. Bombardi, D. G. Porter, F. Baumberger, A. T. Boothroyd, and D. F. McMorrow, Phys. Rev. B 98, 014429 (2018).

[41] I. Collier, R. Hampton, G. Saunders, and A. Stoneham, J. Nucl. Mater. 168, 268 (1989). 